Voix et Images

\title{
La symbolique de l'espace dans les récits de Gabrielle Roy
}

\section{Nicole Bourbonnais}

Volume 7, numéro 2, hiver 1982

Michel Tremblay

URI : https://id.erudit.org/iderudit/200326ar

DOI : https://doi.org/10.7202/200326ar

Aller au sommaire du numéro

\section{Éditeur(s)}

Les Presses de l'Université du Québec

\section{ISSN}

0318-9201 (imprimé)

1705-933X (numérique)

Découvrir la revue

\section{Citer cet article}

Bourbonnais, N. (1982). La symbolique de l'espace dans les récits de Gabrielle Roy. Voix et Images, 7(2), 367-384. https://doi.org/10.7202/200326ar d'utilisation que vous pouvez consulter en ligne.

https://apropos.erudit.org/fr/usagers/politique-dutilisation/ 


\title{
La symbolique de l'espace dans les récits de Gabrielle Roy
}

\author{
par Nicole Bourbonnais
}

Les lecteurs des récits de Gabrielle Roy connaissent sa prédilection pour le paysage imposant des plaines de l'Ouest. Aussi bien dans La Route d'Altamont ${ }^{1}$, où le lecteur est "entraîné en une sorte de traversée d'un infini pays monotone», dans une «plaine fière et solitaire», que dans Ces enfants de ma vie ${ }^{2}$ où "presque tout indice de vie humaine, presque tout détail (rentre) dans le plan infini de la plaine", que dans Le Jardin au bout $d u$ monde ${ }^{3}$ où domine la "plate étendue du pays sans habitation", les silhouettes des personnages se découpent sur un espace qui s'étend à perte de vue.

La nostalgie de l'enfance, la puissance des souvenirs, ne suffisent pas à elles seules à expliquer cette fascination pour les étendues aux confins illimités. On remarque que dans ces récits l'apparition de la plaine s'accompagne presque inévitablement d'une lancinante interrogation sur les mystères de l'existence. Tout se passe comme si l'auteur trouvait dans la plaine nue et rase la matière première à sa rêverie. D'ailleurs, de par sa configuration même, la plaine, espace immense et immuable, n'est-elle pas toute désignée pour servir de symbole universel? Symbole du néant qui guette tout être humain dès sa venue au monde. Symbole aussi de cette vaste Création indifférente où doivent se débattre les habitants de la terre.

Lors d'une enquête littéraire effectuée par Paul Wyczynski ${ }^{4}$, Gabrielle Roy témoigne de l'influence d'une lecture sur son imaginaire, lecture qui conduit tout droit à la contemplation de l'Espace, celle d'une nouvelle de Tchekov intitulée La Steppe :

Longtemps, longtemps, cette lointaine lecture a pénétré mes pensées, m'a façonné, si je puis dire, une manière de voir, de regarder et de saisir le réel. (...) Elle (la steppe) m'était apparue toute pleine de grands paysages plats, un peu dénudés, assez pareils aux plaines du Manitoba que j'avais alors sous les yeux, toute pleine aussi de songeries un peu tristes sur ce que l'avenir peut réserver à chacun, comme si la steppe infinie et l'âme du jeune garçon dont il est question dans cette nouvelle, poursuivaient ensemble la même rêverie. ${ }^{4}$

D'abord sustenté par le paysage familier de l'enfance, l'imaginaire est ensuite réactivé et soutenu par l'activité onirique et par le fantasme de 
l'écriture. Espace onirique donc, mais aussi espace vital dans le sens où l'entend Michel Mansuy dans son étude sur "l'imagination de la vie ":

Si l'imagination (au sens bachelardien du terme) est l'expression en images des instincts fondamentaux, l'imagination de la vie traduit symboliquement le vouloir-vivre dans ses variations multiples : peur et dégoût de vivre, joie de vivre, étonnement de vivre, passion de créer, désir d'éternité. Ainsi, quand l'homme doute de sa vigueur, il voit dans l'inerte une menace de pétrification qui nie de façon intolérable ses droits de vivant à sang chaud. L'iceberg, le rocher nu, le Sahara, l'océan désert matérialisant son incapacité à persévérer dans son être. II est des gens nés pour avoir peur et qui tremblent avant toute raison positive de s'inquiéter. Pour eux, tout est griffe, chaussetrappe ou abîme, l'univers est une hydre ou un gouffre béant. Que, dans une imagination, la peur de vivre se précise en terreur de mourir, on verra se multiplier les images de la décomposition. (...) Mais il arrive que le vouloir-vivre soit plus fort que l'angoisse d'être au monde. L'homme, alors, découvre autour de lui des promesses de renaissance et d'immortalité dans la semence, l'œuf, la chrysalide, même et surtout dans le sarcophage qui, comme un sein maternel, réenfantera le défunt au dernier jour. 5

Ainsi la «steppe manitobaine» qui n'a cessé de réclamer l'invention narrative de Gabrielle Roy éclaire-t-elle une imagination de la vie essentiellement tournée vers la création. II semble que, devant cette démesure et cette vacuité du monde, une sorte de panique et de frénésie soit engendrée. Qu'opposer à cette superbe indifférence et à cette toute-puissance sinon sa propre création, si dérisoire et si transitoire soit-elle? Que ce soit la poupée que la grand-mère confectionne à partir de rien (La Route d'Altamont), ou encore un coin du Québec recréé, embelli, magnifié par la mère de Christine (Rue Deschambault), ou le petit jardin de Martha (Le Jardin au bout du monde), éclatant de couleurs, l'espace humain, lieu de jouissance et de création, travaille à contrer l'espace inhumain, sauvage et insensible de la Création.

Le sentiment d'oppression mais aussi de fascination qu'exerce la plaine sur l'esprit humain est particulièrement sensible dans les récits qui composent le recueil intitulé Le Jardin au bout du monde. Le symbole de l'immigrant comme figure de l'homme exilé sur terre complète bien la métaphore de la plaine originelle. Smouillya, "homme de montagne transplanté dans la plaine nue ${ }^{6}$, se défend contre l'impression d'étouffement ressentie, d'une part, en vivant en reclus, d'autre part, en recherchant la colline qui accroche le regard et rompt l'éternité de l'espace. Les Doukhobors tentent eux aussi désespérément de contrer l'accablante immensité en se laissant prendre au piege de collines imaginaires. Mais nulle part ailleurs que dans la nouvelle éponyme du recueil la valeur métaphorique de l'espace incommensurable, sa place déterminante dans l'imaginaire de l'auteur n'apparaissent avec autant de puissance et d'acuité.

II se passe peu de choses en vérité dans ce récit. Un seul et unique voyage - comme notre vie sur terre - conduit Martha et Stepan, venus 
de la lointaine Ukraine, jusqu'aux confins reculés des terres de l'Ouest canadien, après avoir «traversé un continent, puis l'océan, puis presque tout un autre continent " ${ }^{7}$. Depuis, livrés à eux-mêmes, ils n'ont cessé de se mesurer à ce terrible opposant qu'est la navrante étendue d'un espace monotone.

Semblable en cela à la création à ses débuts, l'espace dans le récit est antérieur aux personnages. Dès l'ouverture du récit, Gabrielle Roy s'applique à rendre sensible, quasi palpable, l'accablante réalité d'un espace illimité :

Plus loin encore que Codessa, sorte de petite capitale ukrainienne dans le Nord canadien, après que l'on a voyagé des heures sur une infinie route de terre, au-delà d'une plaine sauvage apparaissent enfin des signes de ce qui a tenté un jour d'être un village. ${ }^{8}$

D'ores et déjà, les lieux envahissent le texte, reprenant le titre et l'amplifiant. Avec une constance qui ne se démentira pas, l'antinomie perçue entre le "jardin" et le «bout du monde" se maintiendra tout au long de la nouvelle, opposant deux espaces en soi conflictuels mais qui ne seront pas totalement irréductibles l'un à l'autre. Au contraire, ils sont susceptibles de réconciliation car si l'un est immuable et inaccessible, l'autre, tout modeste et transitoire qu'il soit, est proliferant. Sa puissance réside dans son pouvoir de contagion et de répétition.

Ainsi donc, la structure initiale de la nouvelle, divisée en dix chapitres, sert de base au système narratif. En effet, non seulement l'écart perçu dès la première phrase entre un territoire infiniment grand et un autre infiniment petit, sera-t-il inlassablement repris par la suite mais ce paradigme oppositionnel servira de principe structural, produisant de nombreuses ramifications (passif-actif, hostile-chaleureux, éloigné-proche, éterneltransitoire), toutes tributaires d'une dialectique fondamentale: vie-mort, mortel-immortel, humain-divin. En outre, au fur et à mesure que progresse le premier chapitre, le petit village pitoyable qui n'a pu accéder à l'existence est graduellement supplanté par un site, également de dimensions réduites, mais triomphant et euphorique : le jardin multicolore de Martha Yaramko. Comme dans le récit exemplaire, le dénouement est connu à l'avance; le suspense est dédaigné au profit de la leçon ou de l'interprétation. II ne reste plus à la narratrice qu'à reprendre l'histoire à son début en montrant comment s'est opérée la substitution des lieux et des valeurs. II ne lui reste plus qu'à donner le sens de la parabole.

Nous reconnaissons là la manière propre à Gabrielle Roy dans ses "exemplum », celle d'une narratrice qui se dédouble en actrice ou en témoin pour mieux commander l'interprétation. Tel est le cas par exemple de Rue Deschambault ou de Ces enfants de ma vie où derrière le locuteurécrivain se profile la silhouette de Christine enfant ou institutrice. En vertu de cette stratégie de direction, le lecteur est guidé à travers les méandres du récit sans risquer de s'égarer dans des voies d'évitement. A la présence de l'espace qu'est la nature s'ajoute donc la présence de l'écriture comme 
espace avec, comme point d'ancrage, le corps même de la narratrice, témoin oculaire :

Ainsi un jour que m'amenait sur cette route une étrange curiosité - mais plutôt une tristesse de l'esprit, ce goût qui assez souvent m'a prise de découvrir et de partager la plus totale solitude - j'ai vu devant moi, sous le ciel énorme, contre le vent hostile et parmi les herbes hautes, ce petit jardin qui débordait de fleurs. ${ }^{9}$

Détentrice du verbe, du logos, elle constitue le lieu même où se manifeste la métamorphose exemplaire. C'est à travers elle - et son double Martha que s'effectue le renversement de l'espace physique en espace métaphysique. Entre le premier et le dernier chapitre, la figure du chiasme illustre la double transformation salvatrice, comme l'indique le graphique suivant:

\section{Premier chapitre: La plaine : vaste et toute-puissante}

Dernier chapitre: Le jardin, symbole de vie, immortalisé par la volonté de sa créatrice et par l'écriture

Le rapport inversé dans le premier chapitre - l'immensité ayant été réduite à de saisissables dimensions - est donc amplifié dans le chapitre de clôture : le vide de l'éternité est en quelque sorte avalé par la plénitude de l'éphémère. C'est, dans l'espace gulliverien, la victoire de Lilliput sur les géants.

Pendant tout le temps que dure le récit, la narratrice, semblable à une prêtresse qui officie aux rites d'une religion sacrée, prononce inlassablement les paroles litaniques qui édifient, dans un refrain incantatoire, l'architecture du vide et du plein. La narration passe et repasse par les mêmes lieux et les mêmes formules, partageant l'espace en deux : «(...) sous le ciel énorme, contre le vent hostile et parmi les herbes hautes “ce petit jardin qui débordait de fleurs ${ }^{10} \mathrm{~m}$. En vertu de ce mimétisme formel, l'infinie monotonie de la plaine solitaire est mise en relief. En outre, la présence d'un temps jamais terminé mais toujours recommençant se fait tyranniquement sentir. Assez curieusement, redites et récidives, loin de provoquer un piétinement du récit, le font insensiblement avancer, mimant en même temps le mouvement cyclique et répétitif du temps. Une mémoire est ainsi conférée au texte.

D'entrée de jeu, deux espaces président donc aux destinées du récit: l'un inhumain, l'autre, humain. Le premier, de loin le plus imposant car il est cet être-là de toute éternité, se caractérise par deux critères principaux : l'incommensurable et l'incompréhensible. Si son ouverture est symbole de liberté et de découverte, son absence d'être peut aussi attirer dans des abîmes sans fond: 
Cet attrait sur son cœur, à vingt ans, des énormes distances à parcourir, des horizons larges, comment Martha aurait-elle pu encore le comprendre, elle qui en connaissait maintenant l'ennui effroyable. ${ }^{11}$

Leurre donc, mirage, que ces “vastes champs vides ${ }^{12}$ ", que "l'infini silence et la sauvagerie de la plaine ${ }^{13}$ ", que “l'ampleur des horizons inaltérables ${ }^{14}$ " car plutôt que de conduire l'être humain vers le renouveau, la “déconcertante ampleur du ciel et de la terre 15 mystifie et oppresse l'âme. Le "sauvage silence ${ }^{16}$ " paraît étrange à Martha qui lève "au ciel si immensément répandu un regard dérouté ${ }^{17}$ " et qui croit avoir vécu "en des sortes de limbes, entre cette vie et l'Éternel "18. Interminable, la plaine, et le ciel qui la reflète, en viennent tout naturellement à représenter l'éternité inviolable, inclinant “l'homme à se reconnaître le passant d'un jour en ce monde " ${ }^{19}$. Aussi bien dans les «infinis parages d'en haut " 20 que dans “l'infinie plaine de Volhyn» ${ }^{21}$, l'espace pose aux vivants « une sorte d'immense et désespérante interrogation"22. Pourtant, elle est aussi promesse, virtualité qui appelle son incarnation. Cette longue plaine herbeuse est "plongée dans l'attente": elle "s'ouvrait devant leurs yeux pareille a quelque rêverie sans fin sur les hommes et leur destinée "23. Lieu de tous les possibles, la plaine oblige les humains à se définir.

On imagine bien que dans cet espace métaphorisé, quasi mythique, dans ce decor vide et pourtant envahissant, les êtres fictifs qui surgiront auront des préoccupations d'ordre essentiellement métaphysique. Etres en situation, il leur faut «s'attaquer à pareille solitude ${ }^{24}$, tenter de maîtriser l'espace et le temps. Le premier espace humain à être édifié, celui de la foule, ne sera guère plus qu'un lieu commun. Pour appréhender l'énigme de la Création, pour soumettre la plaine, la poignée d'immigrants n'a trouvé mieux que de reproduire les sites historiques conventionnels: l'école et la chapelle. Cette dérisoire tentative de s'approprier l'horizon sauvage s'est révélée inopérante car elle s'en est tenue aux manifestations stéréotypées de la connaissance profane et sacrée. L'école «d'ancien modèle" est abandonnée. Quant à la chapelle, espace sacré qui constitue le recours suprême contre le temps, elle n'est plus que apoussière, ruine, silence!" ${ }^{25}$. Définie par approximations - elle a été construite "comme une église", dotée “d'un semblant de portique», “d'un peu de vitre colorée", des "fentes étroites" lui "servent de fenêtre" 26 -, la chapelle n'a pas d'existence propre. L'intérieur comme l'extérieur de cette «bizarre chapelle" offre la vanité d'un monde sans consistance: toiles d'araignée, odeur de moisi, madone aux traits pâlis témoignent de l'infructueux essai de s'asservir Dieu sur terre. Du moins, par les moyens de l'artifice et de l'apparence. "Qui s'était retiré ?", se demande Martha:

«Dieu, oubliant ses créatures perdues au fond des terres canadiennes? Où eux-mêmes, les êtres humains, par manque d'imagination? (...) Et se pouvait-il que Dieu ne fût aussi qu'un rêve, un désir né de la solitude? 227 
A côté de la foule à l'esprit guère fécond ni inventif, Gabrielle Roy a campé deux protagonistes qui vont incarner les deux tendances opposées de l'imagination de la vie: la peur de vivre et l'ardeur à vivre. Ce sont respectivement Stepan et Martha, couple d'ennemis, d'étrangers intimes quí ne se parlent plus depuis plusieurs années. Ils n'en sont pas moins jumeaux dans leur inlassable recherche du sens de la vie et des signes de Dieu et ils vont à tour de rôle effectuer un rite de passage symbolisé par un déplacement qui leur fournira la réponse voulue pour la conquête de l'immortalité.

Comme issu par scissiparité de la terre inhospitalière et sans fin, Stepan en semble l'émanation maléfique. Pour lui, le monde équivaut à un traquenard. II s'est si bien laissé happer par l'espace hostile qu'il finit par ressembler à une bête sauvage. Ne s'exprimant plus que par des grognements rageurs, indistincts, il passe ses jours loin de toute civilisation «au bout de leur terre", errant dans l'immensité où se perdent les "éclats de (sa) voix maugréante " ${ }^{28}$. Et pour l'attirer, «il n'y avait vraiment plus que l'appel à la nourriture, ainsi que pour une bête de ferme» ${ }^{29}$. Lorsqu'il s'avance « chétive silhouette à courtes jambes et grosse tête embroussaillée», aux "yeux de loup, défiants et sombres", Martha, son épouse, ne peut que se demander avec étonnement si c'était «encore un visage humain " qu'elle «avait sous les yeux? »30.

L'imagination de Stepan toute empreinte de négativisme lui présente la vie comme "un immense exil poudreux" ${ }^{31}$, le porte à ne voir plus «que malignité autour de lui ${ }^{32}$. Sa vision du temps est tout aussi pessimiste: "(..) sa vie lui apparaissait comme un seul long jour monotone " 33 , comme un "long jour aride et venteux»34. Enfoncé dans le passé stérile - il passe ses soirées a lire les vieux numéros du journal ukrainien - il se complaît dans la stagnation et l'immobilisme. En tant que sujet, son champ d'action est restreint; sa compétence, nulle. Outre ses accès de colère «contre cet absurde effort des hommes partout dans le monde pour améliorer leur sort» ${ }^{35}$, il est la plupart du temps «hébété", "somnolent", “à peine conscient", tombant dans "des états de morne stupeur" 36 . Des images lui viennent-elles à l'esprit, elles sont inévitablement évocatrices de discordance et de dégoût : « (...) les yeux égarés des gens qui ont faim et, fouillant l'auge pleine, le goinfre avide des porcs, leurs petites (sic) yeux brillants de contentement ${ }^{37}$.

Enfin n'en pouvant plus « de voir d'autres chérir cette vie que lui eût voulu livrée à l'exécration de tous ${ }^{38}$, il entreprend la rituelle descente aux Enfers, condition essentielle à sa renaissance, qui se présente dans le récit sous la forme d'un voyage vers le lointain village de Codessa. Figure satanique s'il en fât que celle de Stépan, à l'air "dément " "secouant les rênes", avec "des cris rauques" ${ }^{39}$, lançant sa bête à toute allure sur la longue route déserte. Par une sorte d'osmose maléfique, dans la «somnolence de l'esprit ", dans le «brouillard de sa pensée», il s'apparente plus que jamais à l'espace qui l'entoure: (...) «zone de marécages, terres encore plus basses, humides, aux joncs pourrissants, à l'odeur de vase, et que traver- 
sait de loin en loin le cri angoissé des oiseaux $\approx 40$. La contamination est telle “qu'une angoise (sic) traversa en vol rapide les pensées de Stepan " 41. Le présage de la mort prochaine de Martha est inscrit dans le paysage qui transmet à Stepan son sentiment d'angoisse.

Tout en continuant de jouer sur les notions de discordance, Gabrielle Roy fait sentir, toujours par l'intermédiaire de l'espace, l'approche de la métamorphose : «Enfin, il sortit des marais odieux, de leur sifflement d'herbes en lanières, de leur odeur fétide. L'atmosphère s'assainit " ${ }^{2}$. Mais, pour l'instant, Stepan n'éprouve que rancune contre l'injustice qui oppose les «efforts insensés de l'époque pionnière à «cette prospérité, cette aisance, cette belle vie des Ukrainiens d'aujourd'hui " ${ }^{43}$. Il lui faut encore aller jusqu'au fond le plus reculé des Enfers, "s'engouffr(er) dans l'intérieur miobscur " 44 de la taverne, connaître l'ivresse libératrice pour recouvrer l'usage de la parole. Après avoir exprimé sa première crainte, celle que Martha une fois morte aille se plaindre de lui à Dieu, il pose la grande question essentielle: "En fait, y a-t-il quelqu'un pour écouter qui que ce soit? " $45 \mathrm{Ce}$ à quoi le vieux Fédor répond que «Dieu n'écoute pas les vivants " 46 . II doit donc écouter les morts. Ainsi Stepan et Martha arrivent, par des chemins différents, à la même conclusion : Dieu n'existe pas. La nature, la grande Déesse, constituera alors le seul havre possible.

Contrastant avec les imprécations de Stépan et son identification à l'espace infernal, le doux entretien que Martha poursuit inlassablement avec le vent, la lumière, les fleurs, son incessante communion avec la nature, orientent nettement le récit du côté de l'euphorie. Dès sa première apparition, la principale protagoniste est campée dans un décor miniaturisé et intimiste, ce qui, selon Gilbert Durand ${ }^{47}$, signifie une volonté d'inversion de l'hostilité de l'univers et de l'ombre maléfique de la mort:

Tôt ce matin-là, malgré la douleur qui à présent s'éveiliait en son corps en même temps qu'elle, Martha sortit de la basse petite maison chaulée pour venir, en fichu blanc noué sous le menton, son tablier gonflé d'air, reconnaître en son jardin les plantules à peine dégagées de la graine, tout juste perçant à la vie. ${ }^{48}$

Les images d'éclosion, de printemps, de chaumière (isomorphe de la matrice) placent déjà Martha dans un lieu paradisiaque. Même la mort qu'elle porte en elle est traitée avec la même tendresse que ses fleurs bienaimées :

Elle peinait doucement à sa tâche, évitant avec adresse tout mouvement brusque qui eût réveillé la douleur sommeillant pour l'instant dans son flanc droit. Et ainsi avait-elle cet air. de porter en elle quelque chose de précieux. ${ }^{49}$

Volupté de la nature, euphémisation de la mort, douceur du refuge et des recommencements, toutes valeurs qui indiquent une surdétermination de la vie. 
Le personnage féminin n'est guère présenté dans son rôle d'épouse et de mère sinon par de brefs rappels : ses enfants partis depuis longtemps n'écrivent plus et ne viennent plus lui rendre visite; quant à Stepan, son époux, toute communication entre eux a été rompue car ne vivent-ils pas dans des univers, au départ, inconciliables? Le programme narratif de Martha se définit essentiellement dans son rapport avec l'univers créé. En premier lieu, son désir et son savoir orientés du côté de la conquête, n'est-ce pas elle «qui avait souhaité le départ, pesant sur la volonté de Stepan que le long voyage effrayait, elle qui l'avait entraîné par l'ardeur de sa foi en ce pays à découvrir " ? ${ }^{50}$ En second lieu, sa volonté est toute rendue à faire fructifier ses plantes, prodiguant inlassablement ses soins aux fleurs fragiles, si bien qu'elle finit par leur ressembler:

Elle ne se donnait plus qu'à son petit jardin et, ce faisant, tout comme des plantes que l'on entretient, ses pensées aussi se dégageaient du silence et de l'habitude. ${ }^{51}$

Elle est la grande pourvoyeuse, la grande bienfaitrice qui se donne à cette tâche unique, celle de contrer l'hostilité de l'univers :

Pourquoi, se demanda-t-elle, une petite vie aussi douce, aussi tranquille que celle d'une fleur avait-elle tant d'ennemis ? ${ }^{52}$

C'est donc grâce à cette étroite et constante fusion avec la Nature que Martha en vient à oser interroger le ciel, à se laisser aller à «l'étonnement profond de ce qu'est cette étrange vie humaine ${ }^{53}$.

Leur première entreprise “d'apprivoiser la plaine»" 54 , "de créer, devant le silence de Dieu et des hommes, leur petite vie tendre, intime et domestique " 55 s'étant soldée par un échec - l'on songe à la Création d'avant la Chute et au symbolisme de la Chute comme découverte de l'état de mortel - et sentant sa mort venir, Martha effectue à son tour un déplacement rituel pour le passage à l'immortalité. Elle se rend à la chapelle dans une ultime tentative d'y rencontrer Dieu. Contrairement à Stepan, en cours de route, «sur cette longue route de terre, droite et triste, comme si elle venait depuis le commencement des temps $\$ 56$, Martha n'éprouve ni sentiment de dépaysement ni sentiment d'égarement mais «elle se sentait tirée en avant, portée vers une fraternité humaine, une rumeur de voix" et bien qu'elle ait l'impression d'avoir vécu "en bordure du pays, en quelque zone imprécise de vent et de solitude", il lui semble aussi que cette zone "un jour peut-être viendrait à rejoindre le Canada» ${ }^{7}$. Ainsi, l'espoir de Martha, son imagination heureuse et fertile s'exprime-t-elle constamment en termes d'espace et d'avenir.

Après cette impression d'animation et d'aventure, le choc de la chapelle déserte et délabrée est d'autant plus grand. Martha n'y trouve qu'un Dieu absent ou imaginaire. L'espace sacré est inefficace; il faut chercher ailleurs le principe divin :

A supposer qu'il (le Seigneur) fût vivant et présent, elle l'imaginait plus à l'aise au dehors, sur les ailes du vent, dans la fraîcheur de 
l'air, qu'en cette petite chapelle où, quoi qu'elle fît, II subsisterait une odeur de moisi. ${ }^{58}$

Le retour à la nature originelle s'impose. Ses deux éléments représentants seront le vent et le petit jardin de fleurs. Il est d'ailleurs significatif que, la porte de la chapelle à jamais refermee, le "vent aussitôt accouru au ras du sol se remit à la suivre, comme s'il allait aujourd'hui partout avec Martha" 59 .

La substitution de lieux sacrés qui est suggérée, entre la chapelle et le vent, est primordiale puisqu'elle fait du vent le symbole par excellence de la Présence éternelle. Élément dynamique, mobile, provocateur, il règne en despote sur le monde, animant la nature d'un mouvement perpétuel, engendrant d'incessantes mutations, et par là même proclamant la durée :

Tout le pré était couvert de hautes herbes de multiples espèces mais unies, confondues par leur bruissement et leur inlassable flux et reflux. (...) Dans le haut ciel, le vent passait, imprimant aux nuages le même mouvement tranquille qu'en bas aux herbages. ${ }^{60}$

Toujours sensible, toujours attentif, perméable aux influences, il épouse à tour de rôle les divers "mouvements de l'âme, la stérile révolte humaine l'ébranlant presque jusqu'à la folie, les grands coups d'ennui frappant de toutes parts, et aussi l'abandon, la douceur, le calme», mais surtout «il semblait que le vent connût tout cela et tour à tour cherchât à leur donner expression ${ }^{61}$. Traducteur de l'univers, comme l'artiste, comme l'écrivain, non seulement il pénètre les plus intimes replis de l'âme humaine, non seulement il met en communication les êtres et les choses, mais il est la Vie elle-même. II s'oppose à la traditionnelle définition du Principe divin, fixe, immuable, impassible et c'est en cela même qu'il est bénéfique, voire transcendant. N'est-il pas « impétueux et changeant " ${ }^{62}$, sollicité par tout ce qui existe dans l'univers crée, répondant à l'invite de Martha "comme si le son de cette voix humaine (l')eût attiré " ${ }^{63}$, jamais las, ni muet, ni immobile, mais toujours cherchant, toujours emporté par sa foi et son ardeur à découvrir. Comme les fleurs, il figure "une sorte d'enfance éternelle de la création» 64 .

Mais, si c'est en vertu de son extrême labilité que le vent peut connaître la diversité de l'âme humaine et qu'il peut assurer une sorte de réalité palpable du temps, c'est aussi en vertu de cette même labilité qu'il passe brusquement de la douce caresse aux "brûlantes rafales" 65 . Martha se voit alors contrainte de protéger ses fleurs contre celui qui "n'est pas longtemps l'ami des herbes, des fleurs et de l'âme qui aime et soigne la vie "66. Immédiatement après avoir assimilé le vent au Principe éternel, ses deux principaux attributs étant le mouvement et la capacité d'expression, l'auteur lui confère une valeur négative, l'hostilité : il incarne aussi le mal incompréhensible qui sévit sur terre. C'est alors que le personnage de Martha va dominer la scène, supplantant Dieu et sa création angoissante. D'abord, elle porte secours à ses créatures tourmentees tandis que son pendant antinomique avait oublié "ses créatures perdues au fond des terres 
canadiennes " ${ }^{67}$. Ensuite, (épisode immédiatement situé après la visite à la chapelle), la genèse de sa création lui revient en mémoire : elle "se rappela comment était né et avait grandi ce petit jardin, qui était en quelque sorte la véritable histoire de sa vie ${ }^{68}$ ". Voilà le véritable objet poursuivi par la protagoniste, voilà où sa compétence et son savoir - espoir, tendresse, sollicitude - ont été fructueux. "Cette femme qui "se voyait pour ainsi dire sans parents et sans enfants " ${ }^{69}$, sa véritable descendance est celle des fleurs: Martha se perdait "dans le dénombrement à l'infini de la descendance d'une seule fleur", s'émerveillant qu'une seule fleur mise à sécher au plafond" ait pu produire des centaines de roses " 70 . La réussite se situe donc pour Martha non dans l'entreprise de colonisation, non dans son rôle de procréatrice, qui sont des gestes de banale répétition, mais dans l'œuvre de création.

L'analogie entre le chaos de l'univers avant le début de la Création - l'expression temporelle «en ce temps-là " renvoie aux temps nébuleux du chaos - et la terre de Martha est évidente :

C'était le printemps, un immense printemps de boue, de terre presque liquide, de ciel très haut qui se reflétait sur le sol en mille morceaux de bleu décousus. (...) elle avait porté le regard vers la boue, l'infini déroulement de la plaine nue et désolée qui venait de près cerner la cabane. (...) Et c'est alors qu'elle avait eu l'idée de faire porter à cette terre solitaire ses premières fleurs de jardin: des tournesols, peut-être aussi des pavots. ${ }^{71}$

Alors, au sein du chaos et de l'indescriptible naît « une harmonie de couleurs telle qu'immédiatement, elle parut à sa place, tout comme l'horizon, tout comme les nuages ${ }^{72}$. Les fleurs éclatantes se hissent avec jubilation au-dessus de la terre plane. Ce qu'elles perdent en étendue et en duree, elles le gagnent en relief, en éclat et en densité. Cette "masse de couleurs ardentes, qui sautent aux yeux, saisissent l'âme" 73 , cette "masse de fleurs étroitement confondues, mêlant leurs éclatantes couleurs, qui formait comme une ronde serrée et criait l'été à tout l'horizon " 74 font reculer le ciel énorme et humanisent la plaine. L'œil subjugué par ce que leur présence a d'excessif et d'enlevant oublie la terrible menace du néant :'son regard se repose enfin sur un site à sa mesure qui agit comme un baume sur le cœur. Quel réconfort pour le passant et le solitaire qu'est l'être humain en cette vie que l'infinie variété de fleurs $" 75$, la diversité de leurs formes et de leurs couleurs: "pavots écarlates au cœur sombre, d'autres roses, marginés d'une teinte plus accusée», "des lupins sur leurs hampes flexibles, des géraniums aux tons clairs, de fragiles mufliers (...) de hautes fleurs altières, d'autres timides" 76 . Tous les écarts et toutes les invraisemblances se résorbent dans le geste créateur qui insuffle une présence dynamique à l'univers. Un jour, un inconnu sera mystérieusement attiré et consolé «à la vue des tournesols géants et des éclatants pavots" 77 , qui fleurissaient inexplicablement près de la "cabane misérable "78. Pour Gabrielle Roy, l'univers fonctionne, pour peu qu'on le veuille bien, comme un système de vases communicants où l'offrande de l'un agit sur l'autre, où un perpétuel 
échange s'instaure dans la chaîne des humains qui abolit la solitude. L'inconnu n'avait-il pas à son tour offert un paquet de bulbes qui avaient donné "d'innombrables dahlias pourpres" ? ${ }^{79}$ La narratrice sera aussi cette errante au cœur lourd que la vue des fleurs va alléger de sa peine.

Reproduisant la figure du cercle, les fleurs "en ronde serrée" présentent une vision de plénitude et de totalité. "L'espace circulaire", affirme Gilbert Durand, "est plutôt celui du jardin, du fruit, de l'œuf et du ventre, et déplace l'accent symbolique sur les voluptés secrètes de l'intimité " ${ }^{80}$. Rassurant, accessible, il atténue le sentiment de panique engendré par l'immensité spatiale et temporelle, il offre "quelque chose de mesuré, de vulnérable et d'humain (...) dans l'ampleur des horizons inaltérables " ${ }^{81}$. Le jardin pour Gabrielle Roy devient isomorphe du temple ou plutôt il prend la relève, le relayant dans ses fonctions sacrées. Or, de dire Gilbert Durand:

L'espace sacré possède ce remarquable pouvoir d'être multiplié indéfiniment. L'histoire des religions insiste à juste titre sur cette facilité de multiplication des « centres " et sur l'ubiquité absolue du sacré : "La notion d'espace sacré implique l'idée de répétition primordiale qui a consacré cet espace en le transfigurant. "L'homme affirme par là son pouvoir d'éternel recommencement, l'espace sacré devient prototype du temps sacré" 82 .

Promu au rang d'espace sacré, le jardin, minuscule point d'ancrage dans l'infini, est plusieurs fois redoublé dans le récit par d'autres points isolés qui, eux aussi, ont pour effet de découper l'espace, d'en arrêter le déroulement et d'apaiser l'âme. Ainsi, comme la plaine, l'intérieur de la maison de Martha représente l'ennui et la monotonie: au sein de l'uniformité, Martha a introduit "le bleu delphinium", la "couleur jonquille"; elle a orné les vieilles armoires de dessins et recouvert la table d'une «nappe cirée à grosses fleurs rouges sur un fond bleu et jaune " ${ }^{83}$. Ou bien, c'est "l'arbre isolé", le "petit arbre" qui vient rompre «l'intensité de sa solitude» tel «un colporteur dont le sac contenait quelques graines de fleurs rares $" 84$. Autre manifestation ponctuelle et rassérénante que celle du "frêle bois de trembles", rappelant ceux des fermes de Pologne, du "petit bois de trembles (...) tout ruisselant de lumière, avec ses feuilles rebroussées " ${ }^{85}$. Lorsque Stepan, de retour des lieux souterrains, cherchera l'approbation de Martha et l'accueil de la nature, il s'occupera à nettoyer le petit bois et à soigner les fleurs. De nouveau le miracle se produit :

Et le taillis se reprenait à respirer. II réapparaissait peu à peu comme au premier temps de sa vie, pur, jeune, et laissant apercevoir au fond, sous ses branches, le lointain lumineux de la plaine. ${ }^{86}$

Ces oasis de beauté, qui transfigurent le désert de la plaine, ont aussi leur contrepartie dans le temps et dans l'âme. Qu'importe si l'été est si bref, si étrange puisqu'il apporte cette "lumière dorée, ce bien-être de l'air et des feuilles, cette santé de toutes choses, cette ardeur à vivre" ${ }^{87}$, qu'importe la durée éphémère du crépuscule puisqu'elle est cette «poignante illumi- 
nation», "cette incandescence ${ }^{88}$ qui exalte et qui enchante le cœur et l'esprit, qu'importe encore l'ennui de vivre si on peut se souvenir "de curieux et brusques élans de bonheur " ? ${ }^{89}$

Cette capacité infinie de multiplication du jardin sacré finit par l'emporter sur l'étendue. Gagnant peu à peu du terrain, obsédants et envoûtants, ce sont les sites miniatures qui finissent par envahir l'espace. Car tous ces groupes épars, si isolés soient-ils, communiquent inlassablement entre eux, déversant sur les êtres et sur la plaine leur trop-plein de vie. Ainsi, ces soirs de "ciel rouge», «à la fois transitoires et magnifiques", même si “la plaine, à cette heure semblait encore si possible s'élargir", l'âme humaine n'en était que davantage portée à la confiance 90 . Est aboli l'ennui interminable de la plaine désolée lorsque le claquement des feuilles, leur doux bruissement "avait pu faire penser à de la sympathie pour la peine des humains" "1. Lorsque dans "le ciel géant" s'allument les "étoiles si bas", semblables aux "feux de nombreuses maisons", Martha voit «une image d'avenir»: «Se pouvait-il que se peuplât enfin la solitude du monde?" 92 .

En vertu de cette intense circulation entre les éléments naturels, les espaces antinomiques du jardin et de la plaine se rejoignent, contaminés l'un par l'autre, dans un geste d'ultime réconciliation. N'a-t-on pas vu en effet s'adoucir le terrifiant espace, l'inhumain s'humaniser? L'espoir de Martha n'a-t-il pas grandi devant la vision de «la houle ininterrompue des épillets de mil sauvage, la fétuque ciliée, la masse de panics ce grand flot de graminées $\$ 93$ :

Elle eut à nouveau cette pensée que la plaine était absorbée dans un grand rêve de choses à venir, et chantait la patience et la promesse que tout, en temps et lieu, serait accompli. 94

De son côté, le jardin acquiert par osmose valeur d'éternité comme la plaine; il se fond en elle sans s'anéantir et entre à son tour dans la voie mythique. Martha, dont les lancinantes interrogations sur l'existence de l'immortalité et de l'éternité se font de plus en plus pressantes à mesure qu'approche la mort, maintient son refus, à l'heure suprême, d'un au-delà. Les paroles du pope qui "parlait d'espace, d'espace sans limites, incommensurable dans la distance et dans le temps "95 sont devenues caduques. L'immortalité souhaitée par Martha sera à ras de terre: "Mais d'espace infini et de majesté elle en avait eu assez, elle demandait grâce, elle demandait l'oubli " ${ }^{96}$. Sa dernière volonté sera celle d'une acceptation totale de l'ici-bas :

A cette humble immortalité de l'air, du vent et des herbes, elle confia son âme. ${ }^{97}$

La mort ici n'est pas la fin de tout mais un commencement qui nous reconduit au début du récit.

De même que le vide originel est comblé par l'apparition du «paradis terrestre" de Martha, et par ses pouvoirs de multiplication, de même le vide 
de l'oubli qu'entraîne l'état de mortel est aboli par la puissance souveraine de la Parole, elle aussi multiple. Coupée des siens et de son passé, Martha rejoint l'âme de ses ancêtres grâce à un chant d'Ukraine :

Ainsi par quelques bribes de mélodie que retrouvait son souvenir, par quelques paroles lui revenant à l'esprit, elle se sentait rejointe mystérieusement par une âme inconnue d'elle, dont la nostalgique tendresse était toute vivante encore dans ce vieux chant d'Ukraine. ${ }^{98}$

Elle-même demande au vent, doué, on l'a vu, d'une capacité d'expression fort diversifiée, de se souvenir d'elle et aqu'en parcourant le pays, en remuant les herbes, il dise quelque chose de sa vie" ${ }^{99}$. Mais en dernier ressort, l'immortalité est conférée par l'écriture, qui rachète les petites vies ignorées. Grâce à l'instance narratrice, qui assume aussi la fonction de focalisatrice, la modeste existence de Martha ne tombera pas dans l'oubli. D'ailleurs, dans le récit, la délimitation entre le vécu et le narré est très nette: à Martha, la vie, l'accomplissement du quotidien, à la narratrice, les prestiges du récit. Agissant simultanément en témoin et en présentateur (donc dans la paradoxale attitude de la proximité et de l'éloignement), jamais l'instance narratrice n'abandonne sa fonction d'interprète. À toutes ces questions que Martha n'a cessé de se poser, c'est elle qui fournit les réponses. Le discours interieur montre Martha consciente de ses lacunes: "Sans doute eût-il fallu être une autre qu'elle-même pour donner réponse à ces incessantes questions que se posait Martha"100. Or, cette "autre" n'est nulle autre que la passagère d'un jour qui, elle aussi, s'interroge sur le sens de son existence :

En ce temps-là, souvent je me disais : à quoi bon ceci, à quoi bon cela? Écrire m'était une fatigue. Pourquoi inventer une autre histoire, et serait-elle plus proche de la réalité que ne le sont en eux-mêmes les faits? Qui croit encore aux histoires? Du reste toutes n'ont-elles pas été racontées ? 101

L'instance créatrice qu'est Martha lui fournit la réponse : elle écrira pour rendre compte du sens de la vie qui réside avant tout dans sa puissance de creation.

Ce sont la plupart du temps les personnages féminins qui, dans les récits de Gabrielle Roy, sont investis du pouvoir créateur. La filiation établie avec netteté entre la narratrice et Martha avait déjà été suggérée dans le récit intitulé "Ma grand-mère toute-puissante", qui fait partie de La route d'Altamont. Là aussi, la navrante monotonie du paysage, source d'ennui au sens pascalien du terme, conduit l'être ou à périr ou à chercher un palliatif à cette grisaille. La petite Christine, qui devenue adulte et écrivain raconte sa propre histoire, s'ennuie éperdument, seule avec sa grand-mère, surtout au moment du crépuscule:

C'était au moment où le soleil, sur le point de disparaître, jette sur la plaine une grande clarté rouge, lointaine et étrange, qui semble encore la prolonger, et aussi la vider comme de toute présence humaine, la rendre peut-être aux songes sauvages du temps où elle vivait dans sa 
solitude complète. On aurait dit alors que la plaine ne voulait pas sur elle de gens, de maisons, de villages, que, d'un coup, elle eût cherché à se défaire de tout cela, à se retrouver comme autrefois, fière et solitaire. ${ }^{102}$

Ici aussi c'est un paysage primitif, d'avant les Temps, qui aimante le rêve. Ici aussi, c'est la femme qui, par son don d'invention, trouve un divertissement à la mesure de l'ennui : elle "crée» une poupée. Sa petite-fille s'écrie :

Tu es Dieu le Père. Tu es Dieu le Père. Toi aussi, tu sais faire tout de rien. ${ }^{103}$

Plus question de la terrible hérédité de souffrances et de misères qui pèse sur Florentine, Rose-Anna et la vieille Mme Laplante, dans leur rôle de procréatrices, mais d'une connivence bien plus étroite qui les guide du côté de la création. Ce n'est pas non plus notre Père qui est aux cieux mais notre Mère qui a les deux pieds sur terre, qui est l'objet du chœur de louanges, du Magnificat. Dès lors, les images de la nature et de la femme-créatrice se superposent et se confondent pour proclamer la valeur suprême de l'invention. ${ }^{104}$ II n'en va pas autrement dans le récit " Les déserteuses" de Rue Deschambault, où le voyage, l'envers de l'immobilité de la plaine, séduit à tel point la mère de Christine qu'elle décide d'aller revoir le Québec, où les maisons "forment un interminable village" où "de beaux arbres (...) accompagnent les routes". S'y retrouvent les mêmes valeurs de l'intimité que dans Un jardin au bout du Monde :

Ces maisons du Québec, basses, avec d'étroites fenêtres près du sol, de grands toits pointus, n'admettent peut-être pas la lumière du jour autant que nos maisons du Manitoba, mais elles conservent mieux la chaleur des souvenirs. ${ }^{105}$

Or, ce voyage, Éveline l'accomplit dans la transgression, c'est-à-dire sans l'autorisation du Père et, pour renverser les forces négatives du Pouvoir, elle a recours au récit. "Pour cet endroit décisif", explique Gabrielle Pascal, «c'est le pouvoir du verbe qui permet à Éveline de reprendre le contrôle d'une situation perdue ": 106

À la mère de famille décrite comme culpabilisée par son départ s'est substitué un personnage mythique, celui de la conteuse qui, par la puissance de son imagination et les séductions de son talent transcende sa personne et les circonstances. ${ }^{107}$

Parfois, il n'y a que les collines pour animer la plaine et la création se mue en rêverie intérieure, rétablit la filiation entre les humains. Sam Lee Wong aurait-il survécu à l'exil sans une chaîne de petites collines qui arrêtait "la fuite du pays"?

En plaine rase comme était le village, on ne pouvait manquer, en tous temps, d'avoir les yeux fixés sur ces surprenantes collines, et, les retrouvant chaque matin, de retrouver aussi une sorte de refuge contre la sensation de vertige que suscitait, à la longue, la plate immobilité. ${ }^{108}$ 
Les collines comme les mots, mais avec moins de force, jouent un rôle compensateur: sont abolies les cloisons entre les humains, est effacée l'idée de déchéance et de mort. À l'espace inentamable, Gabrielle Roy oppose toute impulsion créatrice.

Une autre nouvelle encore, «De la truite dans l'eau glacée ", présente des pulsions de vie analogues. Visitant les collines de Babcock, l'institutrice, accompagnée du jeune Médéric, sent son âme "s'élargi(r) d'aise et de bonheur " ${ }^{109}$, à la vue cette fois de l'aspect immuable, "du plan infini de la plaine "110. Pourquoi les «innombrables détails captivants" de la plaine, par exemple un coin de «terre fraîchement retournée ", « un infime carré de vert tendre" 111 , ne retiennent-ils pas avant tout son attention? Si la plaine n'est plus oppressante, c'est que l'angle de vision s'est modifié : la jeune fille la contemple d'une certaine hauteur, sur un plateau, situé au faîte des collines. Toujours un élément nouveau, inusité, vient faire la nique à l'ennui. Ici, ce sont les collines qui ont « un caractère révolté " ${ }^{112}$, tandis que dans la nouvelle éponyme de "La Route d'Altamont", «la plaine, depuis le commencement des âges aplanie et soumise, parut se révolter " ${ }^{113}$, et des collines «accoururent de toute part, comme si, délivré de sa pesante immobilité, le pays se mettait en mouvement $" 21$. Le projet de Christine de devenir écrivain viendra supplanter les collines qu'elle n'arrivera plus à retrouver.

A lire ces recoupements, on se rend compte que la nouvelle "Un Jardin au bout du monde " fonctionne comme l'élément-clé du paradigme des récits qui ont la plaine de l'Ouest comme arrière-plan et comme interlocuteur ${ }^{114}$. On peut en effet retrouver dans la plupart de ces récits des variations sur le même thème: le problème de l'existence, symbolisé par l'immensité, le lointain, est résolu dans l'acte de surgissement de l'être prolongé par celui de l'écriture.

1. Gabrielle Roy, La Route d'Altamont, Montréal, HMH, 1969, p. 15-16.

2. Ibid., Ces enfants de ma vie, Montréal, Stanké, 1977, p. 157.

3. Ibid., Le járdin au bout du monde, Montréal, Beauchemin, 1975, p. 154.

4. Paul Wyczynski, "Le Roman canadien-français", Archives des lettres canadiennes, tome III, Ottawa, Fides, 1964, p. 303-304.

5. Michel Mansuy, Études sur l'imagination de la vie, Paris, José Corti, 1970, p. 8.

6. Gabrielle Roy, "Où iras-tu Sam Lee Wong", in Un Jardin au bout du monde, Montréal, Beauchemin, 1975, p. 154.

7. Ibid., p. 154.

8. Ibid., p. 153.

9. Ibid., p. 155.

10. Ibid., p. 155.

11. Ibid., p. 176-177.

12. Ibid., p. 177.

13. Ibid., p. 195.

14. Ibid., p. 198.

15. Jbid., p. 158.

16. Ibid., p. 162. 
17. Ibid., p. 172.

18. Ibid., p. 176.

19. Ibid., p. 177.

20. Ibid., p. 216.

21. Ibid., p. 206.

22. Ibid., p. 216.

23. Ibid., p. 154.

24. Ibid., p. 176.

25. Ibid., p. 176.

26. Ibid., p. 153-154.

27. Ibid., p. 175.

28. Ibid., p. 161.

29. Ibid., p. 163.

30. Ibid., p. 164.

31. Ibid., p. 188.

32. Ibid., p. 188.

33. Ibid., p. 191.

34. Ibid., p. 192.

35. Ibid., p. 165.

36. Ibid., p. 191.

37. Ibid., p. 192.

38. Ibid., p. 193.

39. Ibid., p. 189.

40. Ibid., p. 193.

41. Ibid., p. 194.

42. Ibid., p. 194.

43. Ibid., p. 195.

44. Ibid., p. 196.

45. Ibid., p. 199.

46. Ibid., p. 199.

47. Gilbert Durand, Les Structures anthropologiques de l'imaginaire, Paris, Bordas, 1969, 5 e édition, p. 315-320.

48. Gabrielle Roy, op. cit., p. 157.

49. Ibid., p. 160.

50. Ibid., p. 176.

51. Ibid., p. 159.

52. Ibid., p. 159.

53. Ibid., p. 204.

54. Ibid., p. 177.

55. Ibid., p. 176.

56. Ibid., p. 171.

57. Ibid., p. 172.

58. Ibid., p. 181.

59. Ibid., p. 181.

60. Ibid., p. 180.

61. Ibid., p. 177.

62. Ibid., p. 177.

63. Ibid., p. 178.

64. Ibid., p. 170.

65. Ibid., p. 183.

66. Ibid., p. 183.

67. Ibid., p. 175.

68. C'est moi qui souligne.

69. Ibid., p. 179.

70. Ibid., p. 187.

71. Ibid., p. 184-185.

72. Ibid., p. 185. 
73. Ibid., p. 155.

74. Ibid., p. 169.

75. Ibid., p. 166.

76. Ibid., p. 155-156.

77. Ibid., p. 185-186.

78. Ibid., p. 186.

79. Ibid., p. 186.

80. Gilbert Durand, op. cit., p. 283-284.

81. Gabrielle Roy, op. cit., p. 198.

82. Gilbert Durand, op. cit., p. 284.

83. Gabrielle Roy, op. cit., p. 201.

84. Ibid., p. 205.

85. Ibid., p. 155.

86. Ibid., p. 210.

87. Ibid., p. 169.

88. Ibid., p. 201.

89. Ibid., p. 202.

90. Ibid., p. 201.

91. Ibid., p. 206.

92. Ibid., p. 206.

93. Ibid., p. 180.

94. Ibid., p. 181.

95. Ibid., p. 216.

96. Ibid., p. 216.

97. Ibid., p. 217.

98. Ibid., p. 215-216.

99. Ibid., p. 217. C'est moi qui souligne.

100. Ibid., p. 203.

101. Ibid., p. 155.

102. Gabrielle Roy, "Ma grand-mère toute-puissante", la Route d'Altamont, Montréal, HMH, 1969, p. 15.

103. Ibid., p. 28.

104. A cet égard, il me semble que lorsque Gérard Bessette dans son article «La Route d'Altamont clef de la montagne secrète", conclut à l'assimilation de la colline/montagne à la mère sacrificielle, immolée pour l'Art, il conviendrait d'ajouter à ce symbole celui de la création. La mère n'a-t-elle pas toute sa vie, comme le dit Bessette "dans le pays le plus plat du monde, (...) tenter d'évoquer ses collines originelles" et n'a-t-elle pas aussi "en vivant (sa) vie", donné à sa propre mère "une voix pour s'exprimer (p. 190)". Mais la voix de la mère n'a pas été assez puissante pour susciter le retour permanent des collines ou de l'enfance: elle ne reverra donc jamais ses collines d'autrefois et Christine "perdra" la route d'Altamont. Par contre, la création littéraire viendra prendre la relève. C'est ce qui explique aussi, à mon avis, la joie quasi extatique de Christine a l'approche des collines comme celle de Pierre Cadorai juste avant l'apparition de la montagne. La colline en tant que relief, qu'arrête au sein de la plaine sévère et nue, représente l'inconnu ou encore une réalité transfigurée. Elle préfigure en cela, comme la "voix» de la mère, la magie de la création littéraire. S'il est vrai que la mère est sacrifiée, elle est aussi un guide, un commencement, qui trouvera sa réalisation suprême dans sa fille. On se rend compte alors à quel point cette nouvelle est liée par le symbolisme à la première nouvelle du recueil, "Ma grand-mère toute-puissante ".

105. Gabrielle Roy, "Les déserteuses", Un Jardin au bout du monde, Montréal, Beauchemin, 1975, p. 137.

106. Gabrielle Pascal, “La condition féminine dans l'œuvre de Gabrielle Roy », Voix et Images, vol. V no 1, automne 1979, p. 147.

107. Ibid., p. 148. 
108. “Où iras-tu Sam Lee Wong», Un Jardin au bout du monde, Montréal, Beauchemin, 1975, p. 67.

109. "De la truite dans l'eau glacée", p. 156.

110. Ibid., p. 157.

111. Ibid., p. 157.

112. Ibid., p. 155.

113. Op. cit., p. 202.

114. On peut facilement insérer dans cette série paradigmatique les essais et reportages qui ont été réunis dans le recueil Fragiles lumières de la terre, Montréal, Quinze, 1978. Tiré de Mon héritage du Manitoba, le paragraphe suivant se situe tout à fait dans l'axe symbolique en question :

Mes amours d'enfance, c'est le ciel silencieux de la plaine s'ajustant à la douce terre rase aussi parfaitement que le couvercle sur le plat entier, ciel qui pourrait enfermer, mais qui, au contraire, par la hauteur du dôme, invite à s'élancer, à se délivrer; c'est la silhouette particulière, en deux pans, de nos silos à céréales, leur ombre bleue découpée sur un ciel brouillé de chaleur, seule, par les jours d'été, à signaler au loin les villages de l'immensité plate; ce sont les mirages de ces journées torrides où la sécheresse de route et des champs fait apparaître à l'horizon de miroitantes pièces d'eau qui tremblent à ras.de terre. Ce sont les petits groupes d'arbres, les bluffs assemblés comme pour causer dans le désert du monde, et puis c'est la variété humaine à l'infini (p. 156-157). 